\title{
Atherosclerotic occlusive arterial disease of the lower limbs: a historical account of surgical evolution and vascular reconstruction over thirty four years
}

\author{
P.C.A.Ratnatunga ${ }^{1}$, R. Gunawardene ${ }^{2}$, V Pinto ${ }^{2}$, R. Perera ${ }^{2}$, P.Amerasinghe ${ }^{3}$, P. B. Hewavithana ${ }^{3}$, S.Rosairo ${ }^{3}$, \\ N V I. Ratnatunga ${ }^{4}$ \\ Departments of Surgery ${ }^{1}$, Anaesthesia ${ }^{2}$, Radiology ${ }^{3}$,Pathology ${ }^{4}$. University of Peradeniya and the Teaching Hospital \\ Peradeniya, Sri Lanka.
}

Key words: Chronic ischaemia; atherosclerosis; reconstruction

\begin{abstract}
This is a descriptive study of chronic ischaemia of the lower limb caused by atherosclerotic disease in 588 patients. The study was performed over 34 years in a tertiary clinic in the central province of Sri Lanka. It was found that the pattern of occlusions were similar to that of other reports on western patients. However, critical ischaemia was seen in $57 \%$ of Sri Lankan patients, suggesting a more florid disease. Pioneering efforts of reconstructive surgery in 278 patients and in-hospital results are presented.
\end{abstract}

\section{Introduction}

Historically, the angiographic patterns of lower limb occlusive arterial disease in Sri Lankans has been documented (1). The first documentation of reconstructive arterial surgery in Sri Lanka was by Sheriffdeen, in 1985 (2). In a previous study, our group reported the presentation and natural history of aortic atherosclerosis in Sri Lanka (3). It is essential to distinguish atherosclerotic disease from thromboangiitis obliterans (TAO), which presents in the young, an equally dominant arterial disease of limbs in Sri Lanka, where vascular reconstruction was mostly unrewarding (4). This report is an extended observational study of the presentation of atherosclerosis obliterans (ASO) of the lower limbs in Sri Lanka and our experience of vascular reconstruction. For historic purposes, short comings that we encountered in the early years are documented.

\section{Patients and Method}

Some 1316 patients with vascular claudication or critical ischaemia, i.e. skin ulceration, pre gangrene, or gangrene presenting to the Vascular Clinic of the University Surgical Unit at the Teaching Hospital Peradeniya, Sri Lanka were treated over a period of thirty four years (1974-2007). Of 1316 patients, 588 were evaluated on a prospective basis and form the basis of this report. The study comprises two parts; presen-

\footnotetext{
Correspondence: P.C.A.Ratnatunga

E-mail: channasurg@yahoo.com

Received: 27-05-2017 Accepted: 25-06-2018

(iD) https://orcid.org/0000-0001-5101-892X

DOI: http://doi.org/10.4038/sljs.v36i2.8508
}

tation of atherosclerotic arterial disease of the lower limbs from 1974 to 1993 that was recorded on a prospective basis in 588 patients (group A), and the result of lower limb arterial reconstruction in atherosclerotic occlusive arterial disease in a subset of 278 patients (group B). Records in which data were incomplete were excluded from further study.

ASO was diagnosed if the onset of ischaemic symptoms was later than 50 years. Also, ASO was diagnosed in those with first presentation of symptoms less than 50, if there were primary ASO risk factors (smoking was not weighted), there was evidence of systemic ASO, and in those who did not have features of TAO like upper limb arterial occlusive disease superficial thrombophlebitis in non-varicose veins, were classified as ASO. The diagnosis was reinforced by angiographic, operative appearances and histological data of thrombo-endarterectomy specimens or from dissected vessels of amputated limbs. Reconstructive arterial surgery was performed for claudication that handicapped the patient and for critical ischaemia. Systemic atherosclerosis complicated by ischaemic heart disease and cerebrovascular ischaemic manifestations in patients were comprehensively evaluated before surgery was offered. Morbidity and mortality in hospital was recorded and surgical intervention was considered a success if patients' symptoms regressed and claudication distance improved. In those with critical ischaemia, if foot ulcers healed and when digital and forefoot amputations, which were undertaken for gangrene, had healed enabling limb sparing. All data recorded were subjective, there was no objective evaluation as the concept of a vascular assessment laboratory developed in the latter most part of this audit.

In the early part of our work, lack of timed films on angiography which, at times, resulted in poor visualization of the run off and the lack of prosthetic grafts was a technical handicap. Also, serum lipid profile analysis was not available in our early experience. Patients' data, which included angiographic evidence, were entered into a spread sheet that enabled computerised analysis. Ethical clearance for this study was obtained from the Ethics Committee of the Faculty of Medicine, University of Peradeniya. 


\section{Results}

Group $A$

Patients with ASO were categorized, based on the age of origin of symptoms, as follows; $<35$ years, 36 to 49 and $>50$ years. Based on clinical and angiographic assessment, disease was classified as aorto-iliac (AID), femoro-popliteal (FPD) and tibial arterial disease (TD). Table 1 shows the age at presentation and the level of arterial occlusion based on clinical and angiographic data. Some 334 (57\%) of 588 patients presented with critical ischaemia. Furthermore, a high prevalence of critical ischaemia (46\%), was observed in those $>50$. Tobacco smoking was reported in over 80 percent of our patients across all age groups while hypertension was a co-morbid factor in those young patients, $<35$ years, with atherosclerotic arterial occlusive disease (table 2). Diabetes mellitus, hypertension and hyperlipidaemia were other comorbid features that were seen in our patients. Overall, features of systemic atherosclerosis were seen in 17 percent of patients $<35$ years and up to 21 percent of patients $>35$ years (table 3).

\section{Subset analysis of reconstructive arterial surgery for ASO of the lower limbs-Group B.}

The pattern of lower limb occlusive arterial disease that required reconstruction in 278 patients is shown in table 4 . For clarity, surgical reconstruction has been presented as either aortic and aorto-iliac reconstruction or femoro-popliteal and tibial reconstruction.

\section{Aortic and aorto-iliac reconstruction}

The demography of the group requiring aortic and aorto-iliac reconstruction is presented in table 5. Figures 1 to 4 depict the levels of aortic occlusive disease and indicate the quality of images we obtained in the early years of vascular imaging. Based on angiograms, those with occlusion in the aorto- iliac segment were further classified into juxta renal, sub-renal, distal aorta, not involving the bifurcation, and disease involving the aorta and its bifurcation.

\section{The "run-off" in aortic occlusions}

Analysis of angiograms revealed that the run off was satisfactory in the iliac and/ or common femoral arteries in all of the juxta renal group except in two where there was multi segmental disease, with distal superficial femoral artery patency. In those with low aortic occlusive disease there was a good run off in the iliac arteries except in six of the 31 cases analysed. These patients had an associated distal occlusion- 2 with external iliac and 4 with associated superficial femoral artery occlusion. All patients with critical stenosis of the aortic bifurcation had multi-segment disease- 14 with external iliac artery occlusions and 9 with associated superficial femoral artery occlusions. Reconstructive surgery was possible in all of these patients.

\section{Surgical intervention}

Surgical reconstruction comprised of combinations of aortic replacement by graft, thrombo-endarterectomy and / or venous patch angioplasty. Aortic grafts were either straight grafts or an aorto-bi-femoral prosthetic graft depending on whether disease involved the common iliac arteries. The nonavailability of prosthetic grafts in the early years was the reason we adopted extensive use of thrombo-endarterectomy and vein patch angioplasty. In later years, when grafts became available, graft reconstruction evolved as the dominant procedure (5). Tables 6 and 7 indicate the type of reconstruction and its result. Surgical reconstruction was possible in $182(73 \%)$ of two hundred and forty nine patients with aorto- iliac disease. In the remainder, surgical reconstruction was not possible due to patients not giving consent, non-availability of grafts, associated co-morbidity and gross sepsis of the feet with inguinal lymphadenopathy, despite antibiotics, which precluded use of a graft. It is noteworthy that our results are comparable with other contemporary series $(5,6)$. Complications of aortic reconstruction were; graft to limb embolus that required embolectomy in 5; groin bleed from a false aneurysm in 1, groin wound sepsis -1, leakage of lymph-1 and ventricular ectopics -1. Causes of death in the post-operative period were renal failure in 2 patients, coeliac axis ischaemia and bowel infarction - 1; sudden death in 3 patients, thought to be due to a myocardial cause or intra peritoneal bleed (as post mortems were not undertaken), and in one patient, graft occlusion that presented one year later with critical ischaemia leading to major amputation and death.

\section{Common and external iliac artery reconstruction}

The common iliac segment of the aorto-iliac arterial tree was often associated with aortic bifurcation atheroma though, in some, only the common iliac artery was either stenosed or occluded in isolation (Fig. 5). However, bilateral disease was not uncommon. Although the outflow path was compromised by further extension of an occlusion, a run off was often found downstream as shown in table 8 . The symptomatic limb arteries were invariably reconstructed except in bilaterally symptomatic cases where a bifurcation graft was used for bilateral external iliac artery pathology in 6 patients. The inability to show a run off in 22 cases was chiefly due to technical reasons, since in the early years, "syringe needling" was used to identify patency of the run off in some cases.

In the early years localized thrombo-endarterectomy was performed but in the latter years, if the ankle brachial pressure index (ABPI) of the donor limb was above 0.7 , cross over grafting was performed to preserve the aortic bifurcation area for possible future surgery (7). If the (ABPI) was $<0.7$ in the donor limb, a direct graft was attached to the aorta. If the common femoral artery bifurcation was occluded, which was often present with external iliac artery occlusive disease, a profundoplasty or extended profundoplasty was undertaken 
Table 1. Presentation of patients with occlusive arterial limb disease based on the age group at the onset of symptoms

\begin{tabular}{|l|l|r|r|r|r|r|r|r|r|r|}
\hline $\begin{array}{c}\text { Age group in yrs. ; } \\
\text { AO }(\mathrm{n}=)\end{array}$ & \multicolumn{3}{|c|}{$\begin{array}{c}<35 \\
(\mathrm{n}=36)\end{array}$} & \multicolumn{3}{c|}{$\begin{array}{c}36-49 \\
(\mathrm{n}=112)\end{array}$} & \multicolumn{3}{c|}{$\begin{array}{c}50 \text { and + } \\
(\mathrm{n}=438)\end{array}$} \\
\hline Degree : ischaemia & $\mathrm{C}$ & $\mathrm{Cl}$ & $\mathrm{MA}$ & $\mathrm{C}$ & $\mathrm{Cl}$ & $\mathrm{MA}$ & $\mathrm{C}$ & $\mathrm{Cl}$ & $\mathrm{MA}$ \\
\hline PLO* & $\mathrm{N}=$ & & & & & & & & & \\
\hline AlD & 249 & 3 & 10 & 2 & 21 & 33 & 9 & 109 & 60 & 2 \\
\hline FPD & 253 & 1 & 14 & 2 & 4 & 20 & 2 & 110 & 93 & 7 \\
\hline TD & 86 & 0 & 4 & 0 & 5 & 18 & 0 & 17 & 42 & 0 \\
\hline TOTAL & 588 & 4 & 28 & 4 & 30 & 71 & 11 & 236 & 193 & 9 \\
\hline
\end{tabular}

$\mathrm{C}=$ claudication, $\mathrm{CI}=$ critical ischaemia $\mathrm{MA}=$ major amputation i.e. $\mathrm{AK} / \mathrm{BK}$.

*PLO- Pulse level of occlusion - AID= aortoiliac disease, $\mathrm{FPD}=$ femoro-popliteal disease, $\mathrm{TD}=\mathrm{tibial}$ disease.

Table 2. The prevalence of primary risk factors for atherosclerosis in patients

\begin{tabular}{|l|l|l|l|l|l|l|}
\hline \multicolumn{7}{|c|}{ ATHEROSCLEROTIC RISK FACTORS AMONG PATIENTS WITH ASO } \\
\hline $\begin{array}{l}\text { Age groups } \\
\text { in yrs. (AO) }\end{array}$ & $\mathrm{n}=$ & $\begin{array}{l}\text { Diabetes } \\
\%\end{array}$ & $\begin{array}{l}\text { Hypertension } \\
\%\end{array}$ & $\begin{array}{l}\text { Smoking } \\
\%\end{array}$ & $\begin{array}{l}\text { Hyperlipidaemia } \\
\mathrm{n} / \mathrm{n} \%\end{array}$ \\
\hline$<35$ & 36 & 6 & 71 & 98 & $8 / 29$ & 28 \\
\hline $35-49$ & 112 & 7 & 45 & 98 & $61 / 108$ & 56 \\
\hline$>50$ & 440 & 18 & 40 & 88 & $33 / 61$ & 54 \\
\hline
\end{tabular}

* Number of patients investigated

Table 3. The prevalence of systemic atherosclerosis in patients with occlusive arterial disease of the limbs.

\begin{tabular}{|l|l|l|l|}
\hline \multicolumn{4}{|l|}{ SYSTEMIC ATHEROSCLEROTIC DISEASE - PREVALENCE } \\
\hline Age groups in yrs. (AO) & $\mathrm{n}=$ & $\mathrm{MI} / \mathrm{HF}$ & STROKES \\
\hline$<35$ & 36 & $1 / 3$ & 2 \\
\hline $35-49$ & 112 & $9 / 2$ & 13 \\
\hline$>49$ & 440 & $33 / 17$ & 38 \\
\hline
\end{tabular}

MI: Myocardial Infarction. HF: Heart Failure

Table 4. Overview of the types of lower limb occlusive arterial disease requiring reconstruction.

\begin{tabular}{|l|c|}
\hline Level of Occ: artery & $\mathbf{N}=$ \\
\hline Aortic & 93 \\
\hline Common iliac & 76 \\
\hline External iliac & 13 \\
\hline Common femoral bifurc: & 8 \\
\hline Fem:-Popliteal \& Fem- tibial & 88 \\
\hline Total & 278 \\
\hline
\end{tabular}

Table 5. Demography of 182 patients who underwent aortic or aorto-iliac reconstruction.

\begin{tabular}{|l|l|l|l|l|}
\hline \multicolumn{2}{|l|}{ Aorto-iliac atherosclerotic occlusive disease } & Group B $\mathbf{n = 1 8 2}$ Table 5 \\
\hline DEMOGRAPHY AND PRESENTATION OF AORTO-ILIAC OCCLUSIVE DISEASE \\
\hline & $\begin{array}{l}\text { HIGH AORTIC } \\
\text { OCCLUSION }\end{array}$ & $\begin{array}{l}\text { LOW AORTIC } \\
\text { OCCLUSION }\end{array}$ & $\begin{array}{l}\text { AORTIC BIFURC: } \\
\text { CRITICAL STEN: }\end{array}$ & $\begin{array}{l}\text { COMMON \& } \\
\text { EXTENAL ILIAC } \\
\text { OCC: }\end{array}$ \\
\hline $\mathrm{n}=$ & 32 & 31 & 30 & 89 \\
\hline Mean Age/SD & $48.3 / 10.1$ & $44.8 / 9.2$ & 55.4 .5 & 45.6 \\
\hline M:F & $31: 1$ & $29: 2$ & $26: 4$ & $88: 1$ \\
\hline$\%$ Diabetes & 0 & 0 & 2.6 & 4.4 \\
\hline$\%$ Hypertension & 22.2 & 17.4 & 26.1 & 30.9 \\
\hline$\%$ Hyperlipidaemia & 81.8 & 100 & $?$ & 37.5 \\
\hline$\%$ Claud:/Crit : Ischaemia & $97 / 3$ & $87 / 13$ & $68 / 32$ & $47 / 53^{*}$ \\
\hline
\end{tabular}

Claud: claudication Crit: critical ischaemia 
Table 6. Result of 182 aorto-iliac reconstructions for occlusive atherosclerotic disease

\begin{tabular}{|l|c|c|c|c|c|}
\hline \multicolumn{7}{|l|}{ Reconstructions for ASO aorto-iliac occlusive disease } \\
\hline & $\mathrm{n}=$ & TE & V/Grafts & Good result & Deaths \\
\hline Aortic occlusions & 63 & 4 & 59 & 58 & 4 \\
\hline Aorto-iliac critical stenoses & 30 & 18 & 12 & 25 & 3 \\
\hline $\begin{array}{l}\text { Aortic ASO with Common } \\
\text { Iliac occlusions }\end{array}$ & 76 & 37 & 39 & 62 & 3 \\
\hline External -iliac Occlusions & 13 & 3 & 10 & 7 & 2 \\
\hline
\end{tabular}

* Indicates unknown outcome in some patients.

TE- Thrombo-endarterectomy; V/Graft: Vein or graft patch angioplasty or graft bypass

Table 7. Type of aortic reconstruction and result in 93 patients

\begin{tabular}{|ll|l|l|l|l|l|}
\hline \multicolumn{6}{|c|}{ Levels of Aortic atherosclerotic Occlusions and their Reconstructions } \\
\hline Level of occlusion & Number & TE+PA & Grafts & Success & Death \\
\hline Juxta renal (JRAO) & Fig. 1 & 31 & 0 & ABF-31 & $29^{*}$ & 1 \\
\hline Sub renal (SRAO) & Fig. 2 & 01 & 0 & ABF-1 & 1 & 0 \\
\hline Low aortic (LAO) Fig. 3 & 31 & 7 & ABF-24 & 28 & 3 \\
\hline Aorto-iliac critical stenosis (AICS) Fig. 4 & 30 & 21 & ABF-9 & $25^{*}$ & 3 \\
\hline Totals & 93 & 28 & ABF-65 & 83 & 7 \\
\hline
\end{tabular}

*Indicates that in some patients outcome data were not available.

TE+/-PA - Thrombo-endarterectomy patch angioplasty; ABF- Aorto-bifemoral prostheses.

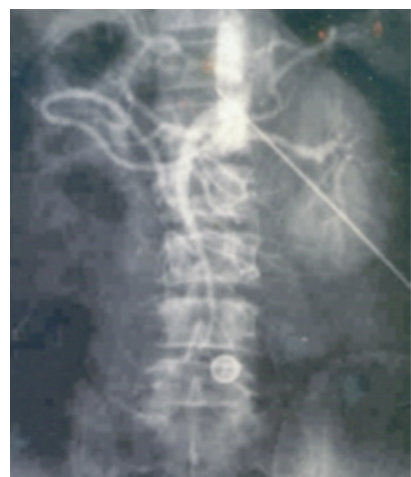

Figure 1. Juxta renal stenosis

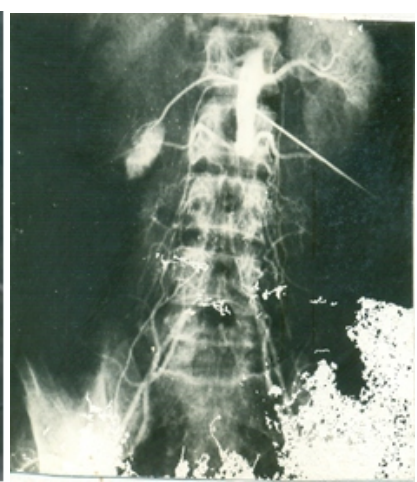

Figure 2. Sub-renal

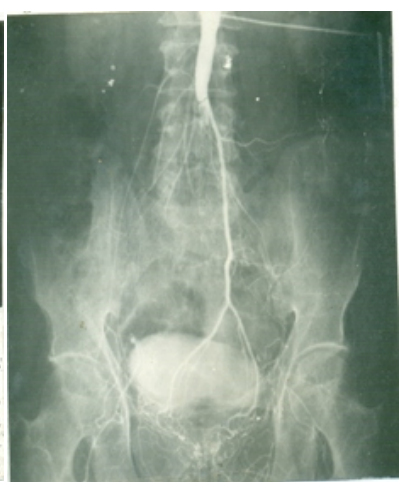

Figure 3. Distal aortic

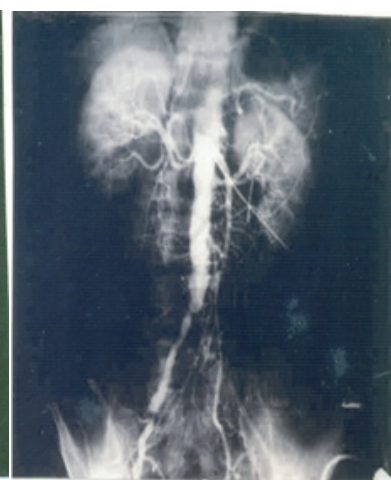

Figure 4. Aortic

Figures 1 to 4. Images of angiography undertaken in patients with occlusive arterial disease secondary to atherosclerosis indicating the levels of aortic involvement.

Table 8. The pattern of run-off in 76 limb reconstructions for common and external iliac artery disease

\begin{tabular}{|l|l|l|}
\hline Level of patent arterial & Bilateral & Unilateral \\
segment & & \\
\hline Bifurcation & 3 & 5 \\
Common femoral & 8 & 20 \\
Common femoral & 8 & 5 \\
Mid superficial femoral & 1 & 3 \\
Distal superficial femoral & 8 & 15 \\
No run off & - & - \\
\hline & 28 & 48 \\
\hline
\end{tabular}


comprising thrombo-endarterectomy with a patch of vein or prosthetic graft - table 9. Complications of common iliac and external iliac reconstruction included, abandonment of the procedure because of a grossly calcified aorta and iliac vessel complex, post-endarterectomy vessel occlusion leading to below knee (BKA) and subsequently an above knee (AKA) amputation in 2 patients; one died of renal failure and another due to myocardial infarction. Other complications included iliofemoral graft occlusion requiring embolectomy -1 , false aneurysm in 1, adhesive intestinal obstruction -1 and inguinal wound sepsis.

\section{Femoro-popliteal and tibial artery reconstruction}

\section{Common femoral reconstruction}

In general, surgical reconstruction for infra-inguinal arterial disease was confined to disease of the femoral artery and that of the arteries in the proximal part of the leg. Reconstructive surgical intervention was not undertaken at that time for distal tibial artery and distal vessel occlusion. Instead, these patients were treated by non- operative methods, such as a program to increase claudication distance, reduction in smoking, control of co-morbid disease such as hypertension and diabetes and, if required, distal amputation. Surgical reconstruction of the common femoral artery in isolation that was undertaken for critical ischaemia, owing to ease of access, in our experience, yielded poor results (table 10). This was mostly due to florid downstream disease; six of 8 reconstructive procedures, all of who had multi-segment disease and diabetes, were followed by graft or vessel occlusion leading to major amputations and one death from myocardial infarction. Graft sepsis was a problem and treated with bactericidal antibiotics.

\section{Reconstruction for femoro-popliteal segment athero-sclerotic disease}

The femoro-popliteal segment was found to be the site most frequently obstructed (Fig. 6). Angiographic analysis of the run-off at the femoro-popliteal occlusion was possible in 74 patients. The data revealed that in Table 11, 38 patients the high popliteal, supra geniculate segment was patent, the lower, infra-geniculate segment was patent in 19, and that there was no visualisation of a run-off in 17. The result is probably due to timing constraints at angiography in the early years. Thus we had to resort to operative exploration and intra-operative angiographic evaluation of the popliteal artery to determine distal arterial patency. When patency of the popliteal artery was established intra operatively, the patient underwent femoro-popliteal reconstruction with prior consent. In the rest, where distal popliteal artery patency could not be established by intra-operative assessment, the procedure was abandoned and details not recorded. For historical purpose, we report that localized popliteal artery reconstructions were undertaken in a limited number of patients in the early years, which yielded poor results (table 11). In the early part of our work, and in all infra-geniculate procedures, we used reverse saphenous vein grafts to reestablish flow. Synthetic graft (GORE-TEX, Arizona, USA. was used after 1990 in a proportion of patients, who had no evidence of infection, and who required supra-geniculate arterial bypass graft surgery. Post-surgical complications in this group included, vein graft occlusion in 3 patients - two received synthetic grafts and the third underwent below knee amputation. Infective groin lymphadenopathy, common in those with critical ischaemia and lower limb infections, that did not respond to antibiotics, often risked graft infection in the proximal groin anastomosis. This was, to an extent, mitigated by the lateral approach to the femoral artery (8). Other complications encountered were lymphatic leaks and post-surgical myocardial infarction.

\section{Management of tibial arterial disease}

In 86 patients, there was isolated tibial disease and these patients mostly presented with critical ischaemia (table 12). As previously mentioned, management in this group of patients centred on non-operative measures and lumbar sympathectomy or amputation.

\section{Discussion}

There is no substantial report of surgical reconstruction for atherosclerotic arterial disease of the lower limbs in Sri Lanka. This study is both a report of surgical evolution for ischaemic lower limb arterial surgery over 34 years, and presents previously unknown data in Sri Lankan patients with arterial ischaemia of the lower limbs.

An incidence of critical ischaemia in 57 percent of Sri Lankan patients with atherosclerotic occlusive disease is dramatically different from reports from the United Kingdom $(6,9)$. There may be an association with florid atherosclerosis that is, in general, reported in the coronary arteries of South Asians (10). In part, this phenomenon has been attributed to the small diameter of coronary arteries in South Asians (10). This begs the question, "Is this phenomenon the lower limb artery equivalent in South Asians?" Another hypothesis is that atherosclerotic arterial disease occurs in vessels previously primed by thrombo-angiitis obliterans, as has been reported in Israel (11). Such high prevalence may also represent late presentation in our patients.

In the early period of our work, thrombo-angiitis obliterans was the dominant disease in Sri Lanka, for which arterial reconstruction was often unsuccessful (3). We observed that the prevalence of atherosclerotic lower limb arterial disease gradually became more dominant over TAO, where in the latter two decades of this study, there was a reduction in the presentation of TAO patients, which made surgical reconstruction more feasible. Corresponding to this change in aetiology, we undertook fewer lumbar sympathectomy and lower limb amputations and more reconstruction procedures for lower limb ischaemia. 


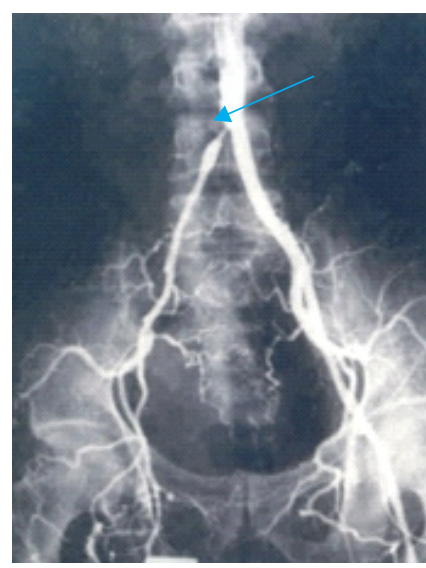

Figure 5. Aorto-iliac angiogram indicating unilateral atherosclerotic disease of the iliac artery (arrow)

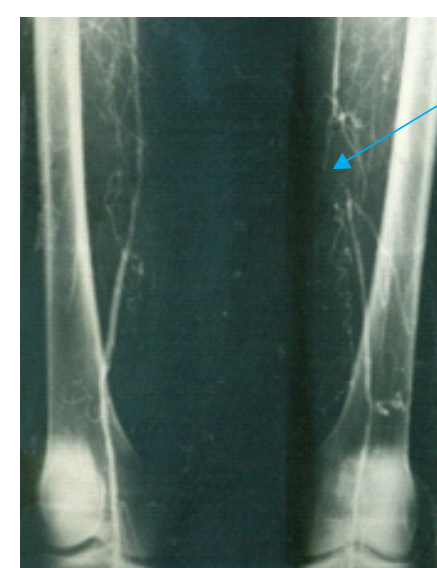

Figure 6. Femoral angiogram showing the most frequent site of occlusion at the femoro-popliteal segment (arrow)

Table 9. Result of reconstruction for symptomatic iliac artery atherosclerosis

\begin{tabular}{|l|c|l|l|l|l|l|l|}
\hline \multicolumn{7}{|c|}{ Reconstructions for ASO iliac disease } \\
\hline & Number & TE/VGA & FFGCO & A/I FGBP & ABF & Success & Death \\
\hline Common iliac & 76 & 37 & 13 & 22 & 4 & 62 & 3 \\
\hline External iliac & 13 & 3 & 4 & 4 & 2 & 7 & 2 \\
\hline
\end{tabular}

TE/VGA - Thrombo-endarterectomy and vein or graft patch angioplasty;

FFCO - Femoro-femoral cross over graft/vein bypass;

A I/FGB - Aorto/ ilio-femoral graft bypass; ABF - Aorto-femoral graft bypass.

Table 10. Outcome of common femoral artery reconstruction for atherosclerosis at its bifurcation (CFB)

\begin{tabular}{|l|c|c|c|c|c|c|}
\hline \multicolumn{7}{|c|}{ Common Femoral Bifurcation (CF B) Atherosclerotic Disease $\mathbf{n = 8}$} \\
\hline & Number & CFTE & CFTEV/GPA & EFCOGBP & Success & Death \\
\hline CFB & 8 & 2 & 5 & 1 & 2 & 1 \\
\hline
\end{tabular}

CFTE- Common femoral thrombo-endarterectomy;

CFTE- VGPA common femoral thrombo endarterectomy and vein or graft patch angioplasty; EFCOGBP- Femoro-femoral crossover graft bypass with CFTE.

Table 11. Outcome of common femoral artery reconstruction for atherosclerosis at its bifurcation (CFB)

\begin{tabular}{|l|l|l|l|l|l|l|l|l|}
\hline \multicolumn{9}{|c|}{ Femoro- popliteal atherosclerotic Disease } \\
& & Number & $\begin{array}{l}\text { Supragen } \\
\text { FPV/GBP }\end{array}$ & $\begin{array}{l}\text { Infragen } \\
\text { FPV/GBP }\end{array}$ & F-TBP & $\begin{array}{l}\text { TE+/- } \\
\text { V/GPA }\end{array}$ & Success & Death \\
\hline \multirow{3}{*}{ Femoro-popliteal } & $\begin{array}{l}\text { Early } \\
1974-1989\end{array}$ & 38 & 27 & 6 & 1 & 4 & 25 & 2 \\
\cline { 2 - 11 } & $\begin{array}{l}\text { Late } \\
1990-2007\end{array}$ & 38 & 11 & 13 & 14 & 0 & 31 & 0 \\
\hline Localised popliteal & & 12 & & & & 10 & 3 & 0 \\
\hline Total & & 88 & 38 & 19 & 15 & 14 & 59 & 2 \\
\hline
\end{tabular}

FPV/GBP - Fem-popliteal bypass graft using either vein or graft $\quad$ F-TBP - Fem-tibial bypass Infragen - Infra-geniculate

TE/+/-VP/GPA - Thrombo-endarterectomy and vein/graft patch angioplasty Supragen - Supra-geniculate 
Table 12. Demography of patients with occlusive tibial and distal artery disease

\begin{tabular}{|l|l|l|l|}
\hline \multicolumn{4}{|c|}{$\begin{array}{c}\text { Demography of Tibial and distal occlusive } \\
\text { disease }\end{array}$} \\
\hline Age (years) & $<35$ & $35-49$ & $>50$ \\
\hline Number & 4 & 23 & 59 \\
\hline Female & 1 & 4 & 22 \\
\hline Non-smoker (\%) & 2 & 6 & 14 \\
\hline Diabetes & 1 & 2 & 22 \\
\hline $\begin{array}{l}\text { Ischaemic heart } \\
\text { disease }\end{array}$ & 2 & 4 & 6 \\
\hline Stroke & 1 & 1 & 11 \\
\hline $\mathrm{C} / \mathrm{Cl}$ & $0 / 4$ & $5 / 18$ & $17 / 42$ \\
\hline$\% \mathrm{Cl}$ & 100 & 78 & 71 \\
\hline
\end{tabular}

C- Claudication only; CI- Critical ischaemia +/- claudication

The aortic occlusions were mostly in male patients, of younger age and in those with hyperlipidaemia, although serum analysis for hyperlipidaemia became routine in the latter years of the study. Somehow, juxta renal aortic occlusive disease seemed to protect the arterial tree downstream from occlusive atherosclerosis, and in this series, 97 percent of patients with aortic disease had only claudication and 87 percent with distal aortic involvement presenting with claudication of the lower limbs. These data are similar to reports from countries with a dominant Caucasian population (8). For reconstruction, this is an advantage, as post-operative graft sepsis is a risk in those who present with critical ischaemia and either associated overt or sub-clinical infection in the lower extremities. By contrast, atherosclerotic disease of the aortic bifurcation and distal arteries seemed to be a presenting feature in mainly older adult patients, who also had a greater percentage of critical ischaemia at presentation. Older patients also tended to have multi-segment disease and a greater prevalence of diabetes compared with their younger counterparts, which reduced the success of outcome of surgical reconstruction. Included in this group were four females probably falling into the "small aortas in small women syndrome (12). Patch aortoplasty was quite successful in this group.

Overall, mortality in our study was $6.6 \%$ and was comparable to reports of 7\% (5) and 6.3\% (9), from other centres. As is established, the commonest site of occlusion of the femoropopliteal segment of the artery was at the adductor hiatus in this study and in most international data (13). Data of femoropopliteal reconstruction for limb ischaemia in the '90 s showed that results of femoro-popliteal bypass procedures which did incorporate vascular grafts that crossed the knee were superior to the use of infra-geniculate graft procedures (14). As surgical techniques and imaging modalities have further evolved to enable surgeons undertake distal vascular reconstruction, and yet further, to use minimally invasive techniques in limb re-vascularisation, further reports of results in these areas would be awaited with interest. This study falls short in its longer-term follow up of graft patency and multivariate analysis of surgical outcomes related to age, risk factors for ASO, and gender. In conclusion, this crosssectional study over 34 years reports a process of surgical evolution for lower limb ischemia in a population where the primary pathology has changed from thrombo-angiitis obliterans to predominantly atherosclerosis that is similar to the West. Athrosclerosis induced stenosis of the distal aorta, which involved the iliac arteries, showed greater prevalence of critical ischaemia at presentation, which indicated multisegment disease. The latter differs from the West.

All authors disclose no conflict of interest. The study was conducted in accordance with the ethical standards of the relevant institutional or national ethics committee and the Helsinki Declaration of 1975, as revised in 2000 .

\section{References}

1. Weerasena M. Peripheral Arterial disease in Sri Lanka. Ceylon Medical Journal, 1976; 22: 159-176.

2.Sheriffdeen A, H. Peripheral Arterial Surgery. Ceylon Medical Journal, 1985; 30: 13-27.

3.Ratnatunga C. Hewavithane B.H. Atherosclerotic Occlusive Disease of the Aorta, Clinical Aspects. Sri Lanka Journal of Medicine, 2001; 10:25-36.

4. Shionoya S, Ban I, Nakata T, Matsubara J, Hirai M, Miyazaki A. Vascular Reconstruction in Buerger's Disease. British Journal Surgery, 1976; 63: 841-846.

https://doi.org/10.1002/bjs.1800631102

5. Minken S L, De Weese J A, Southgate W A, Mahoney E B, Rob C G. Aortoiliac reconstruction in atherosclerotic occlusive disease. Surgery, Gynecology and Obstetrics, 1968; 126: 1056-1060.

6. Irvine W T, Booth R A D, Myers K. Arterial Surgery for aorto-iliac occlusive disease. Early and late results in 238 patients. Lancet, 1972; vol: $738-741$.

7. Fahal AH, Mc Donald P, Marston A. Femoro-femoral bypass in unilateral iliac occlusion. British Journal of Surgery; 1989; 76: 2225.https://doi.org/10.1002/bjs.1800760108

8.Sheriffdeen A.H. A new approach to the femoral artery. Ceylon Medical Journal, 1984; 29: 93-96.

9.Taylor G W. and Calo A R. Atherosclerosis of arteries of lower limbs. British Medical Journal, 1962; 1: 507-510. https://doi.org/10.1136/bmj.1.5277.507

10.Ghumman M. Coronary Disease in South Asians. Clinical Correlations -N Y U Langone online journal of Medicine 2009; accessed 18.7.2018.

11.Mozes M, Cahansky G, Doitsch V, Adar V. The association of atherosclerosis and Buerger's disease. A Clinical and Radiological Study. Journal of Cardiovascular Surgery. 1970; 11: 52-59

12.Cronenwett JL, Davies J.T, Gooch JH,Garett L.E. Aorto- iliac disease in women. Surgery 1980; 88: 775-784.

13.Mavor G E. The pathology and the management of chronic ischaemia of the lower limb. Journal of the Royal College of Surgeons Edinburgh 1958; 3:264-285.

14.Veith F J, Gupta S.K., Ascer E, White-Flores S, Samson R.H, Scher L A, et al six year prospective multicentre randomized comparison of autologous saphenous vein and expanded polytetrafluoroethylene grafts in infra inguinal arterial reconstruction. Journal of Vascular Surgery 1986; 3: 104-114. https://doi.org/10.1016/0741-5214(86)90073-X 